\title{
Osteotomia de nivelamento do plato da tíbia
}

\section{Tibial plato leveling osteotomy}

\author{
Angelica Cecilia Tatarunas ${ }^{1 *}$; Steven A. Martinez ${ }^{2}$; Julia Maria Matera ${ }^{3}$
}

\section{Resumo}

A osteotomia do platô da tíbia (TPLO) é um tratamento relativamente novo e inovador para a ruptura do ligamento cruzado cranial (RLCC) na espécie canina. Ao invés de restaurar a função do ligamento, o procedimento promove estabilidade funcional para a articulação do joelho, por eliminar ou neutralizar a força tibial cranial durante a sustentação de peso. A proposta do presente estudo é revisar a técnica de TPLO, enfatizando o procedimento, técnica cirúrgica, cuidados pós-operatórios e complicações. A técnica da TPLO consiste na realização de uma osteotomia circular do platô da tíbia com rotação de sua porção caudal até a obtenção do ângulo desejado. Após o nivelamento do platô da tíbia, placa e parafusos são utilizados para estabilizar a osteotomia até que ocorra a consolidação óssea. Complicações associadas com o procedimento incluem fratura da tuberosidade da tíbia e tendinose do tendão patelar. O procedimento vem se tornando bastante popular para o tratamento cirúrgico da RLCC em cães de raças grandes, porém os efeitos em longo prazo ainda não estão completamente elucidados e tem sido demonstrado que a técnica de TPLO não coíbe a doença articular degenerativa.

Palavras-chave: Ligamento cruzado cranial, osteotomia de nivelamento do platô da tíbia, joelho

\begin{abstract}
The tibial plateau leveling osteotomy (TPLO) is a relatively new and innovative surgical treatment for the cranial cruciate ligament rupture in the canine species. The real intent of the procedure is to provide functional stability to the stifle joint by eliminating or neutralizing the cranial tibial thrust during weight bearing instead to restore the cranial cruciate ligament function. The proposal of this study is to report a review of the TPLO procedure, emphasizing procedure, surgical technique, post operative care and complications. The TPLO procedure consists in a radial osteotomy in the tibial plato and rotation of the caudal plateau in order to obtain a desired angle. After the leveling of the tibial plateau, a bone plate and screws are used to stabilize the osteotomy until bone is healed up. The complications that have been associated with the procedure include tibial tuberosity fracture and patellar tendon tendinosis. This procedure has become increasingly more popular for surgical treatment of cranial cruciate ligament injuries in large breed dog. The long term clinical results have not been completely elucidated yet. It has been showed that this technique doesn't halt the degenerative joint disease.
\end{abstract}

Key words: Cranial cruciate ligament, tibial plato leveling osteotomy, stifle

1 Pós-Doutoranda do Departamento de Cirurgia da Faculdade de Medicina Veterinária e Zootécnica da Universidade de São Paulo, SP, Brasil. E-mail: angelvet@usp.br.

2 Professor Associado do Departamento de Cirurgia Ortopédica de Pequenos Animais da Washington State University, WA, EUA.

3 Professora Titular do Departamento de Cirurgia da FMVZ/USP, SP, Brasil.

* Autor para correspondência 


\section{Introdução}

A ruptura do ligamento cruzado cranial (RLCC) é afecção que acomete com freqüência o joelho do cão, sendo considerada causa prevalente de doença articular degenerativa desta articulação (JOHNSON; JOHNSON, 1993; HAYASHI et al., 2003; PIERMATTEI; FLO; DeCAMP, 2006). A ruptura ocorre quando forças articulares excedem a força de tensão do ligamento íntegro ou enfraquecido por degeneração crônica (VASSEUR, 2003). O diagnóstico baseia-se principalmente na detecção da presença de instabilidade articular, utilizando-se os testes de gaveta e compressão tibial. Porém, a ausência de instabilidade não exclui a doença (VASSEUR, 2003; PIERMATTEI; FLO; DeCAMP, 2006). O tratamento cirúrgico é de eleição, e apesar das várias técnicas (intra ou extra-articulares) descritas para a sua reparação (WARZEE et al., 2001; PIERMATTEI; FLO; DeCAMP, 2006), nenhuma delas mostrou resultado satisfatório suficiente para comprovar superioridade em relação as demais técnicas quanto ao retorno a função normal do membro e prevenção de doença articular degenerativa (ARNOCZKY; MARSHALL, 1977; WARZEE et al., 2001; KOWALESKI; MCCARTHY, 2004; ARAGON; BUDSBERG, 2005; CONZEMIUS et al., 2005).

A fim de sobrepujar estas limitações e baseandose em recentes conceitos de biomecânica articular, o desenvolvimento das técnicas de osteotomia proximal da tíbia deu iniciou a um novo período para o tratamento da RLCC na espécie canina. Diferente dos procedimentos intra ou extra-articulares que tem por objetivo estabilizar o joelho por restringir o movimento da tíbia, as técnicas de osteotomia da tíbia proximal são projetadas para neutralizar a força tibial cranial ou thrust tibial cranial (TTCr) (SLOCUM; DEVINE, 1983; SLOCUM; SLOCUM, 1993; SLOCUM; DEVINE-SLOCUM, 1998; WARZEE et al., 2001).

A técnica de osteotomia de nivelamento do platô da tíbia ou tibial plato leveling osteotomy (TPLO) foi proposta por Slocum e Devine (1983), e consistia na realização de uma osteotomia em cunha na tíbia proximal. Esta foi subseqüentemente modificada para uma osteotomia circular, com rotação do platô da tíbia caudal e distalmente e fixação do fragmento ósseo com placa e parafuso (SLOCUM; SLOCUM, 1993). A técnica foi patenteada pela Slocum Enterprises, com o objetivo de padronização e conseqüente melhora dos resultados. A patente da técnica expirou em Julho de 2004, permitindo maior divulgação de seu aprendizado (STAUFFER et al., 2006).

\section{Revisão de Literatura}

Apesar dos inúmeros estudos sobre a RLCC em cães, a sua exata patogênese ainda não é totalmente entendida (JOHNSON; JOHNSON, 1993; HAYASHI et al., 2003). Cães com membros pélvicos retos, estreitamento do sulco intercondilar ou demasiada angulação do platô da tíbia são considerados predispostos (PIERMATTEI; FLO; DeCAMP, 2006). Enquanto no homem o platô da tíbia possui uma inclinação caudal de aproximadamente $10^{\circ}$, no cão este valor é cerca de $20^{\circ}$ a $30^{\circ}$. Como conseqüência, as forças compressivas e a estabilidade articular são diferentes entre as espécies. No joelho do homem as forças compressivas resultam em aumento da estabilidade articular, enquanto que no cão estas forças promovem a translação cranial da tíbia em relação aos côndilos do fêmur (SLOCUM; DEVINE, 1983; LI; GIL; KANAMORI, 1999). Durante a fase de apoio do ciclo do andar, ou seja, etapa de sustentação de peso as forças de reação ao solo juntamente com as forças musculares, geram forças compressivas ao longo da tíbia. Visto o platô da tíbia do cão possuir uma inclinação caudal e distal, tal compressão gera uma força orientada cranialmente chamada força cranial da tíbia ou thrust tibial cranial (TTCr), que induz a translação cranial da tíbia em relação aos côndilos do fêmur (HENDERSON; MILTON, 1978).

Técnicas previamente descritas (over-the top ou a sutura fabelo tibial lateral) para a reparação 
do LCCr no cão têm por objetivo estabilizar a articulação durante a fase passiva do ciclo do andar, já a TPLO visa anular o TTCr durante a etapa de apoio do membro (SLOCUM; DEVINE-SLOCUM, 1998; LINEBERGER et al., 2005). Como a TPLO promove uma estabilização dinâmica e não estática da articulação o teste de gaveta permanecerá positivo, mas não o teste de compressão tibial (PIERMATTEI; FLO; DeCAMP, 2006).

O ângulo do platô da tíbia (APT) varia entre os indivíduos, sendo que a conformação esquelética do cão pode influenciar o seu valor (MORRIS; LIPOWITZ, 2001; WILKE et al., 2002). Estudos mostram que os cães portadores de RLCC têm um APT maior do que os cães sem a doença (READ; ROBINS, 1982; SLOCUM; DEVINE, 1983; MORRIS; LIPOWITZ, 2001), embora este dado tenha sido questionado recentemente em estudo realizado por Wilke et al. (2002).

A técnica da TPLO originalmente propõe a obtenção de um APT final de $5^{\circ}$. Não é necessário e nem indicado nivelar o platô da tíbia a $0^{\circ}$ para se obter a estabilidade funcional desta articulação (SLOCUM; DEVINE, 1983). Warzee et al. (2001) realizaram estudo biomecânico e concluíram que o ângulo de $6,5^{\circ}$ é suficiente para este objetivo. Após a realização da TPLO o ligamento cruzado caudal (LCCd) se torna o estabilizador primário da translação crânio caudal do joelho, pois a estabilização ativa é obtida pela conversão do TTCr em força tibial caudal ou thrust tibial caudal (TTCd). Portanto, assim como o maior ângulo na porção caudal do platô da tíbia seja associado com a RLCC em cães, o excessivo aumento do ângulo na porção cranial do platô da tíbia pode predispor o LCCd a lesão (WARZEE et al., 2001; REIF; HULSE; HAUPTMAN, 2002).

A TPLO é realizada em quatro etapas: determinação pré-operatória do APT, osteotomia da tíbia proximal, rotação do segmento de platô da tíbia e fixação interna da osteotomia. A exata determinação do APT é essencial, pois quantifica a rotação do platô da tíbia para a obtenção de um APT de $5^{\circ}$ (SLOCUM; SLOCUM, 1993; KOWALESKI; MCCARTHY, 2004). O APT é mensurado a partir de radiografia em projeção lateral, com o centro do feixe de raios-X na articulação do joelho, com a tíbia paralela ao chassi e incluindo a articulação tíbiotársica (VASSEUR, 2003; GRIERSON et al., 2005; PIERMATTEI; FLO; DeCAMP, 2006). Desenha-se inicialmente uma linha unindo os ápices cranial e caudal do côndilo medial da tíbia, e a seguir o eixo da tíbia é estabelecido unindo-se o centro do platô da tíbia, que consiste no ponto médio entre os tubérculos intercondilares e o centro da articulação talocrural. O APT é, então, definido entre a linha que une os ápices cranial e caudal do côndilo medial da tíbia e uma linha desenhada perpendicularmente àquela do eixo da tíbia (DEJARDIN, 2003; VASSEUR, 2003) (Figura 1).

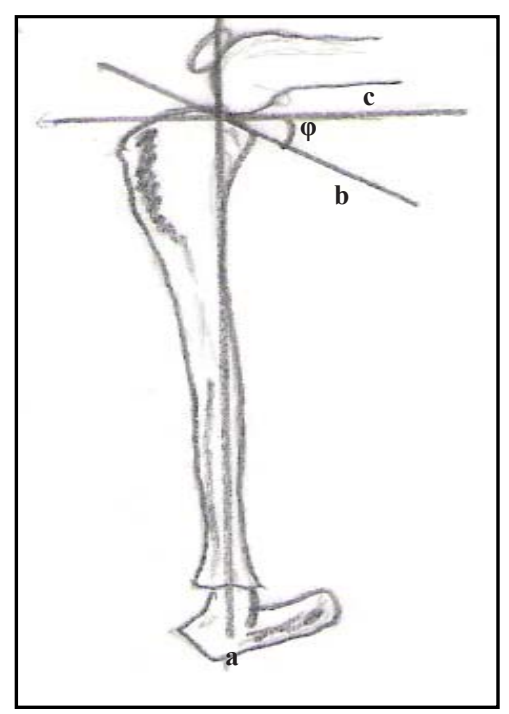

Figura 1. Diagrama demonstrando mensuração do angulo do platô da tíbia. A linha vertical (a) representa o eixo funcional da tíbia e une o centro da do tubérculo intercondilar da tíbia com o centro do talus; a linha do platô da tíbia (b) une as margens cranial e caudal do platô da tíbia; o ângulo do platô da tíbia $(\varphi)$ é medido a partir de uma linha (c) perpendicular ao eixo funcional da tíbia e que passa sobre a linha do platô tibial (b).

Pesquisadores têm manifestado preocupação com a possibilidade de ocorrer variação na determinação do valor do APT. O incorreto posicionamento da 
tíbia durante o exame radiográfico e a presença de osteoartrose pode induzir mensuração errônea do APT, devido às alterações das referências anatômicas. Segundo Reif et al. (2004), o posicionamento inadequado do membro pode alterar o APT em até $3,6^{\circ}$, sendo sugerido a superposição acurada dos côndilos do fêmur e da tíbia durante a projeção radiográfica. A experiência do observador para a mensuração do APT também deve ser considerada (FETTIG et al., 2003; REIF et al., 2004; GRIERSON et al., 2005).

Embora tenha sido demonstrado em estudo in vitro que um APT abaixo de $6,5^{\circ}$ foi suficiente para eliminar completamente o TTCr durante a sustentação de peso do membro (WARZEE et al., 2001), o impacto clínico desta informação ainda permanece obscuro. $\mathrm{O}$ valor do APT obtido a partir de projeção radiográfica lateral é utilizado para determinar em milímetros o grau necessário de rotação do platô da tíbia. São disponíveis tabelas com os valores de conversão previamente calculados (SLOCUM; DEVINE, 1983; SLOCUM; SLOCUM, 1993; SLOCUM; DEVINE-SLOCUM, 1998).

\section{Técnica Cirúrgica}

Anteriormente à reparação do ligamento pela TPLO as estruturas intra-articulares devem ser avaliadas por artrotomia ou artroscopia. O menisco medial tem que ser especialmente inspecionado para a presença de lesão, e os resquícios de LCCr quando presentes devem ser removidos (PIERMATTEI; FLO; DeCAMP, 2006).

É realizada extensa tricotomia do membro a ser operado, desde a região da articulação coxofemoral até a sua extremidade. $\mathrm{O}$ animal é posicionado em decúbito dorsal de modo a permitir amplo movimento das articulações. A TPLO é iniciada com incisão cutânea na face medial da articulação do joelho, a qual é estendida até a região média proximal da tíbia. Após divulsão do tecido celular subcutâneo, as inserções dos músculos grácil e semitendinoso e a bainha caudal do músculo sartório são identificadas e seccionadas. O ligamento colateral lateral é identificado. A fim de facilitar a orientação da osteotomia pode-se utilizar equipamento (jig) especialmente desenhado para a TPLO, o qual é disposto no plano sagital da face medial da tíbia (SCHMERBACH et al., 2007). O osso é osteotomizado com serra e lâmina circular e rotacionado até obtenção do ângulo de $5^{\circ}$ (SLOCUM; DEVINE-SLOCUM, 1998). Estudos in vitro demonstraram que a correta posição da osteotomia foi importante para a obtenção do APT adequado (KOWALESKI; MCCARTHY, 2004; KOWALESKI et al., 2005). Durante a realização da osteotomia os tecidos moles periarticulares e o ligamento patelar devem ser protegidos. Evita-se o superaquecimento ósseo durante a osteotomia por constante irrigação com salina. Seqüencialmente, o fragmento de platô tibial osteotomizado é rotacionado até a obtenção do APT desejado e em seguida fixado com placa especialmente desenhada para o procedimento e parafusos ortopédicos (SLOCUM; DEVINE-SLOCUM, 1998) (Figura 2).

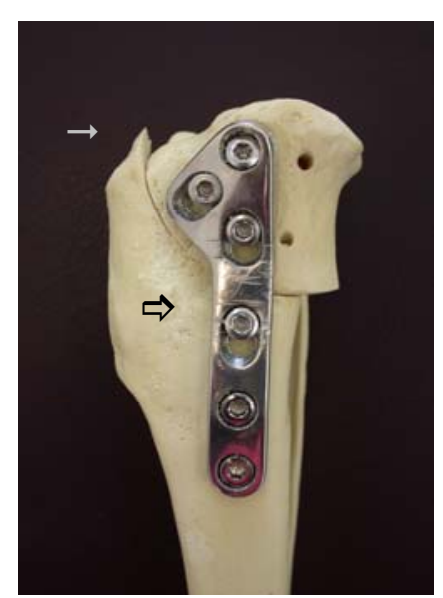

Figura 2. Modelo demonstrando a articulação do joelho após a realização da osteotomia de nivelamento do platô da tíbia $(\rightarrow)$ e fixação com placa e parafusos $(\Rightarrow)$

Deve-se fazer avaliação radiográfica (lateral e caudocranial) imediatamente após o término da cirurgia, a fim de certificar o alinhamento ósseo, posição do implante e da osteotomia em relação ao espaço articular (SLOCUM; DEVINE-SLOCUM, 1998). 
Meniscos são estruturas intra-articulares importantes para a manutenção da estabilidade articular, transmissão da carga e propriocepção (JOHNSON et al., 2004; BRIGGS, 2004). A TPLO é um procedimento cirúrgico que predispõe o menisco medial à lesão. A TPLO reduz, mas não elimina o deslocamento da tíbia em relação aos côndilos do fêmur, portanto, translação cranial residual pode predispor o pólo caudal do menisco medial a lesão por compressão cíclica repetitiva promovida pelo côndilo do fêmur e platô da tíbia (SLOCUM; SLOCUM, 1993; KENNEDY et al., 2005). Em conformidade com o modelo biomecânico do joelho do cão, na ausência do LCCr o corno posterior do menisco medial passa a atuar como restritor passivo primário da translação cranial da tíbia em relação aos côndilos do fêmur, aumentando portanto, as chances de ocorrência de lesão durante o reposicionamento da tíbia para o apoio do membro (THOMPSON; FU, 1993).

A liberação do menisco medial ou meniscal release, que é efetuada concomitante com a TPLO, tem por finalidade tentar preservar a integridade do menisco e, conseqüentemente, a sua função (SLOCUM; SLOCUM, 1993; KENNEDY et al., 2005). A realização da técnica é contraditória entre os pesquisadores (SLOCUM; SLOCUM, 1993; SLOCUM; DEVINE-SLOCUM, 1998; POZZI et al., 2006). Teoricamente, a secção do ligamento menisco tibial (liberação axial) ou a secção radial do corpo do menisco, justamente caudal ao ligamento colateral medial (liberação abaxial), possibilita a movimentação do corno caudal do menisco medial para além do côndilo do fêmur, enquanto ocorre a translação cranial da tíbia em relação aos côndilos do fêmur. Portanto, com o reposicionamento crâniocaudal da tíbia durante a fase de apoio do membro, o corno medial do menisco pode ser protegido de trauma proveniente do fêmur. Kennedy et al. (2005) realizaram estudo comparando as formas axial e abaxial de liberação do menisco medial e concluíram que ambas pouparam o pólo caudal do menisco medial, porém observaram que a liberação abaxial exibiu deformação residual do pólo caudal do menisco, sugerindo poder ser fonte de dor.

\section{Cuidado pós-operatório}

Os cuidados no pós-operatório visam o controle da dor e a restrição de atividade até que ocorra a consolidação óssea (HOELZLER et al., 2005). É indicado a realização de caminhada com uso de guia nos três primeiros meses de pós-operatório, seguido de aumento gradual e progressivo. As reavaliações clínicas e radiográficas geralmente são realizadas com 6 a 8 semanas de pós-operatório, tempo geralmente suficiente para que ocorra a consolidação óssea. Em estudo realizado por Ballagas et al. (2004), cães que tiveram seu LCCr seccionado e foram subseqüentemente submetidos a reparação pela técnica de TPLO apresentaram retorno normal a função do membro avaliado por placa de força com 18 semanas de pós-operatório. Semelhante as demais cirurgias articulares, um programa de reabilitação está indicado a fim de otimizar e acelerar a recuperação do membro.

\section{Complicações}

Complicações trans e pós-operatórias ocorrem em $18 \%$ a $28 \%$ das articulações submetidas a TPLO (PRIDDY et al., 2003; PACCHIANA et al., 2003; STAUFFER et al., 2006). A literatura cita quebra da broca, pino ou parafuso, fratura de cabeça da fíbula, tíbia ou patela, laceração da vasculatura poplítea e hemorragia, colocação de parafuso ou pino intra-articular, infecção, falha de implante, fístula, seqüestro, avulsão da tuberosidade da tíbia com e sem deslocamento e inflamação do tendão patelar, não consolidação óssea e/ou o retardo da mesma (PRIDDY et al., 2003; PACCHIANA et al., 2003; BARNHART, 2003; STAUFFER et al., 2006) e luxação do tendão extensor longo dos dedos (HAELAND; SJÔSTRÔM, 2007). Em estudo realizado sobre a ocorrência de avulsão da tuberosidade da tíbia após a TPLO, Kergosien et al. (2004) concluíram que a sua presença, sem deslocamento perceptível ao exame radiográfico, não era clinicamente significante, pois não comprometeu a evolução pós-operatória e nem resultou em 
deslocamento ósseo. Ainda, segundo estes autores, um fragmento remanescente da tuberosidade da tíbia de maior espessura foi mais resistente à fratura. Carey et al. (2005) observaram espessamento moderado a severo do tendão patelar em exame radiográfico após dois meses de pós-operatório de TPLO, sendo que cerca de $7 \%$ dos cães portadores de espessamento severo, desenvolveram tendinose patelar. De acordo com Priddy et al. (2003) e Kergosien et al. (2004), a realização da TPLO em ambas as articulações em um mesmo procedimento anestésico pode gerar maior índice de complicações.

Estudos clínicos têm revelado que o APT final obtido com a realização da TPLO tem sido diferente do ângulo de $5^{\circ}$ inicialmente proposto por Slocum e Slocum (1993) como sendo o ideal para se obter resultado satisfatório com a utilização da técnica. Robinson et al. (2006) estudaram a cinética da locomoção em Labradores Retrievers portadores de RLCC submetidos a TPLO e concluíram que o cão pode apresentar recuperação satisfatória do membro com ampla margem de valores de APT. Moeller, Cross e Rapoff (2006) demonstraram que o valor do APT aumentou após a consolidação do fragmento de platô da tíbia osteotomizado, apesar da adequada fixação óssea. As conseqüências da alteração do APT para valores diferentes do originalmente sugerido $\left(5^{\circ}\right)$ para a TPLO ainda não são conhecidas.

\section{Considerações Finais}

A RLCC no cão é uma doença complexa, sendo que vários fatores são considerados predisponentes ou desencadeantes como genética, mecanismos imune-mediados, raça, idade, sexo, castração, obesidade, sulco intercondilar, biomecânica local e ângulo do platô da tíbia.

Os conceitos que foram desenvolvidos juntamente com a técnica de TPLO expõem nova dimensão sobre o tratamento desta doença na espécie canina. Acredita-se que a técnica não substitui completamente aquelas anteriormente descritas e que alguns questionamentos ainda devem ser esclarecidos com as pesquisas.

Dada a própria complexidade da RLCC no cão e o número de fatores que podem estar envolvidos com a doença, provavelmente caberá ao cirurgião decidir qual o melhor tratamento a ser empregado para cada animal, de acordo com a provável etiopatogenia em cada indivíduo.

A TPLO promove uma nova abordagem para o tratamento da RLCC, porém constitui um procedimento cirúrgico relativamente elaborado, de custo alto e as complicações devem ser consideradas. Apesar da grande divulgação de seu emprego, principalmente nos Estados, algumas importantes considerações ainda precisam ser elucidadas.

\section{Agradecimentos}

À Fundação de Amparo e Pesquisa do Estado de São Paulo (FAPESP)

\section{Referências}

ARAGON, C. L.; BUDSBERG, S. C. Applications of evidence-based medicine: cranial cruciate ligament injury repair in the dog. Veterinary Surgery, Philadelphia, v. 34, n. 2, p. 93-98, 2005.

ARNOCZKY, S. P.; MARSHALL, J. L. The cruciate ligaments of the canine stifle: an anatomical and functional analysis. Journal of the American Veterinary Medical Association, Schaumburg, v. 38, n. 11, p. 18071814, 1977.

BALLAGAS, A. J.; MONTGOMERY, R. D.; HENDERSON, R. A.; GILLETTE, R. Pre- and postoperative force plate analysis of dogs with experimentally transected cranial cruciate ligaments treated using tibial plateau leveling osteotomy. Veterinary Surgery, Philadelphia, v. 33, n. 2, p. 187-190, 2004.

BARNHART, M. D. Results of single-session bilateral tibial plateau leveling osteotomies as a treatment for bilaterally ruptured cranial cruciate ligaments in dogs: 25 cases (2000-2001). Journal of the American Animal Hospital Association, Lakewood, v. 39, n. 6, p. 573-578, 2003.

BRIGGS, K. K. The canine meniscus: injury and treatment. The Compendium on Continuing Education for the Practicing Veterinarian, Princeton, v. 26, n. 9, p. 687-697, 2004. 
CAREY, K.; AIKEN, S. W.; DIRESTA, G. R.; HERR, L. G.; MONETTE, S. Radiographic and clinical changes of the patellar tendon after tibial plateau leveling osteotomy. Veterinary and Comparative Orthopaedics and Traumatology, Stuttgart, v. 18, n. 4, p. 235-242, 2005.

CONZEMIUS, M. G.; EVANS, R. B.; BESANCON, M. F.; GORDON, W. J.; HORSTMAN, C. L.; HOEFLE, W. D.; NIEVES, M. A.; WAGNER, S. D. Effect of surgical technique on limb function after surgery for rupture of the cranial cruciate ligament in dogs. Journal of the American Veterinary Medical Association, Schaumburg, v. 226, n. 2, p. 232-236, 2005.

DEJARDIN; L. M. Tibial plateau leveling osteotomy. In: SLATTER, D. (Ed.) Textbook of small animal surgery. 3.ed. Philadelphia: Saunders, 2003. p. 2133-2143.

FETTIG; A. A.; RAND, W. M.; SATO, A. F.; SOLANO, M.; MCCARTHY, R. J.; BOUDRIEAU, R. J. Observer variability of tibial plateau slope measurement in 40 dogs with cranial cruciate ligament-deficient stifle joints. Veterinary Surgery, Philadelphia, v. 32, n. 5, p. 471-478, 2003.

GRIERSON, J.; SANDERS, M.; GUITAN, J.; PEAD, M. Comparison of anatomical tibial plateau angle versus observer measurement from lateral radiographs in dogs. Veterinary and Comparative Orthopaedics and Traumatology, Stuttgart, v. 18, n. 4, p. 215-219, 2005.

HAYASHI, K.; FRANK, J. D.; DUBINSKY, C.; ZHENGLING, H.; MARKEL, M. D.; MANLEY, P. A. A. V.; MUIR, P. Histologic changes in ruptured canine cranial cruciate ligament. Veterinary Surgery, Philadelphia, v. 32, n. 3, p. 269-277, 2003.

HAELAND, P. J.; SJOSTROM, L. Luxation of the long digital extensor tendon as a complication to tibial plateau leveling osteotomy. A presentation of four cases. Veterinary and Comparative Orthopaedics and Traumatology, Stuttgart, v. 20, n. 4, p. 224-226, 2007.

HENDERSON, R. A.; MILTON, J. L. The tibial compression mechanism: a diagnostic aid in stifle injuries. Journal of the American Animal Hospital Association, Lakewood, v. 14, n. 6, p. 474-479, 1978.

HOELZLER, M. G.; HARVEY, R. C.; LIDBETTER, D. A.; MILLIS, D. L. Comparison of perioperative analgesic protocols for dogs undergoing tibial plateau leveling osteotomy, Veterinary Surgery, Knoxville, v.5, n. 34, p. 337-344, 2005.

JOHNSON, K. A.; FRANCIS, D. J.; MANLEY, P. A.; CHU, Q.; CATERSON, B. Comparison of the effects of caudal ple hemi-meniscectomy and complete medial meniscectomy in the canine stifle joint. The American Journal of Veterinary Research, Chicago, v. 65, n. 11, p. 1053-1060, 2004.
JOHNSON, J. M.; JOHNSON, A. L. Cranial cruciate ligament rupture. Pathogenesis, diagnosis, and postoperative rehabilitation. Veterinary Clinics of North America: Small Animal Practice, Philadelphia, v. 23, n. 12, p. 717-733, 1993.

KENNEDY, S. C.; DUNNING, D.; BISCHOFF, M. G.; KURIASHKIN, I. V.; PIJANOWSKI, G. J. The effect of axial and abaxial release on meniscal displacement in the dog. Veterinary and Comparative Orthopaedics and Traumatology, Stuttgart, v. 18, n. 3, p. 227-234, 2005.

KERGOSIEN, D. H.; BARNHART, M. D.; KEES, C. E.; DANIELSON, B. G.; BROURMAN, J. D.; DEHOFF, W. D.; SCHERTEL, E. R. Radiographic and clinical changes of the tibial tuberosity after tibial plateau leveling osteotomy. Veterinary Surgery, Philadelphia, v. 33, n.5, p. 468-474, 2004.

KOWALESKI, M. P.; MCCARTHY, R. J. Geometric analysis evaluating the effect of tibial plateau leveling osteotomy position on postoperative tibial plateau slope. Veterinary and Comparative Orthopaedics and Traumatology, Stuttgart, v. 17, n. 1, p. 30-34, 2004.

KOWALESKI, M. P.; APELT, D.; MATTOON, J. S.; LITSKY, A. S. The effect of tibial plateau leveling osteotomy position on cranial tibial subluxation: an in vitro study. Veterinary Surgery, Philadelphia, v. 34, n. 4, p. 332-336, 2005.

LI, G.; GIL, J.; KANAMORI, A. A validated threedimensional computational model of a human knee joint. Journal of Biomechanical Enggineering, New York, v. 121, n. 6, p. 657-662, 1999.

LINEBERGER, J. A.; ALLEN, D. A.; WILSON, E. R.; TOBIAS, T. A.; SHAIKEN, L. G.; SHIROMA, J. T. Comparison of radiographic arthritic changes associated with two variations of tibial plateau leveling osteotomy. A retrospective clinical study. Veterinary and Comparative Orthopaedics and Traumatology, Stuttgart, v. 18, n. 1, p. 13-17, 2005.

MOELlER, E. M.; CROSS, A. R.; RAPOFF, A. J. Change in tibial plateau angle after tibial plateau leveling osteotomy in dogs. Veterinary Surgery, Philadelphia, v. 35, n. 5, p. 460-464, 2006.

MORRIS, E.; LIPOWITZ, A. J. Comparison of tibial plateau angles in dogs with and without cranial cruciate ligament injuries. Journal of the American Veterinary Medical Association, Schaumburg, v. 218, n. 3, p. 363366, 2001.

PACCHIANA, P. D.; MORRIS, E.; GILLINGS, S. L.; JESSEN, C. R.; LIPOWITZ, A. J. Surgical and postoperative complications associated with tibial plateau leveling osteotomy in dogs with cranial cruciate ligament 
rupture: 397 cases (1998-2001). Journal of the American Veterinary Medical Association, Schaumburg, v. 222, n. 2, p. 184-193, 2003.

PIERMATTEI, D. L.; FLO, G. L.; DeCAMP, C. E. The stifle joint, In: Brinker, piermattei, and flo's handbook of small animal orthopedics and fracture repair. 4.ed. Philadelphia: Saunders, 2006. p. 562-660

POZZI, A.; KOWALESKI, M. P.; APELT, D.; MEADOWS, C.; ANDREWS, C. M.; JOHNSON, K. A. Effect of medial meniscal release on tibial translation after tibial plateau leveling osteotomy. Veterinary Surgery, Philadelphia, v. 35, n. 5, p. 490-494, 2006.

PRIDDY, N. H.; TOMLINSON, J. L.; DODAM, J. R.; HORNBOSTEL, J. E. Complications with and owner assessment of the outcome of tibial plateau leveling osteotomy for treatment of cranial cruciate ligament rupture in dogs: 193 cases (1997-2001). Journal of the American Veterinary Medical Association, Schaumburg, v. 222, n. 12, p. 1726-1732, 2003.

READ, R. A.; ROBINS, G. M. Deformity of the proximal tibia in dogs. Veterinary Record, London, v. 111, n. 13, p. 295-298, 1982.

REIF, U.; HULSE, D. A.; HAUPTMAN, J. G. Effect of tibial plateau leveling on stability of the canine cranial cruciate-deficient stifle joint: an in vitro study. Veterinary Surgery, Philadelphia, v. 31, n. 2, p. 147-154, 2002.

REIF, U.; DEJARDIN, L. M.; PROBST, C. W.; DECAMP, C. E.; FLO, G. L.; JOHNSON, A. L. Influence of limb positioning and measurement method on the magnitude of the tibial plateau angle. Veterinary Surgery, Philadelphia, v. 33, n. 4, p. 368-375, 2004.

ROBINSON, D. A.; MASON, D. R.; EVANS, R.; CONZEMIUS, M. G. The effect of tibial plateau angle on ground reaction forces 4-17 months after tibial plateau leveling osteotomy in labradro retrievers. Veterinary Surgery, Philadelphia, v. 35, n. 3, p. 294-299, 2006.

SCHMERBACH, K. I.; BOELTZIG, C. K. M.; REIF, U.; WIESER, J. C.; KELLER, T.; GREVEL, V. In vitro comparison of tibial plateau leveling osteotomy with and without use of a tibial plateau leveling jig. Veterinary Surgery, Philadelphia, v. 36, n. 2, p. 156-163, 2007.
SLOCUM, B.; DEVINE-SLOCUM, T. Meniscal release. In: BOJRAB, M. J.; ELLISON, G. W.; SLOCUM, B. (Ed.) Current techniques in small animal surgery. 4.ed. Baltimore: Williams \& Wilkins, 1998. p. 1197-1199.

SLOCUM, B.; DEVINE, T. Cranial tibial thrust: a primary force in the canine stifle. Journal of the American Veterinary Medical Association, Schaumburg, v. 183, n. 4, p. 456-459, 1983.

SLOCUM, B.; SLOCUM, T. D. Tibial plateau leveling osteotomy for repair of cranial cruciate ligament rupture in the canine. Veterinary Clinics of North America: Small Animal Practice, Philadelphia, v. 23, n. 4, p. $777-$ 95, 1993.

STAUFFER, K. D.; TUTTlE, T. A.; ELKINS, A. D.; WEHRENBERG, A. P.; CHARACTER, B. J. Complications associated with 696 tibial plateau leveling osteotomies (2001-2003). Journal of the American Animal Hospital Association, Lakewood, v. 42, n. 1, p. 44-50, 2006.

THOMPSON, W. O.; FU, F. H. The meniscus in the cruciate-deficient knee. Clinical Journal of Sports Medicine, New York, v. 12, n. 6, p. 771-796, 1993.

VASSEUR, P. B. Stifle joint. .In: SLATTER, D. Textbook of small animal surgery. 3.ed. Philadelphia: Elsevier Science, 2003. p. 2090-2133.

WARZEE, C. C.; DEJARDIN, L. M.; ARNOCZKY, S. P.; PERRY, R. L. Effect of tibial plateau leveling on cranial and caudal tibial thrusts in canine cranial cruciate deficient stifles: an in vitro experimental study. Veterinary Surgery, Philadelphia, v. 30, n. 3, p. 278-286, 2001.

WILKE, V. L.; CONZEMIUS, M. G.; BESANCON, M. F.; EVANS, R. B.; RITTER, M. Comparison of tibial plateau angle between clinically normal greyhounds and labrador retrievers with and without rupture of the cranial cruciate ligament. Journal of American Veterinary Medical Association, Schaumburg, v. 221, n. 10, p. 1426-1429, 2002. 